\title{
Conocimiento y práctica del diseño en la formación de profesionales en áreas no concurrentes del diseño en universidades públicas de Bogotá, Colombia
}

\section{Knowledge and practice of design in the training of professionals in non-concurrent areas of design in public universities in Bogotá, Colombia}

\author{
Isabel Cristina Agudelo-Torres ${ }^{1}$ \\ Jimmy Leonardo Velasco-Sabogal²
}

Recibido: abril 05 de 2018

Aceptado: junio 28 de 2018

\section{Resumen}

En el presente artículo de investigación se aborda la necesidad de establecer la estructura, el conocimiento y la práctica de lo entendido como actividades de diseño, desde sus dimensiones: pensamiento, método y estética, dentro de áreas de enseñanza universitaria no asociadas a profesiones de Diseño. Se busca identificar cómo se entienden y ejecutan procesos de enseñanza en torno a la resolución de problemas débilmente estructurados en otras áreas del conocimiento y en contextos académicos de formación superior, en universidades públicas de Bogotá, Colombia. Para ello, se desarrolla una labor de descripción, caracterización y análisis de los contenidos curriculares que siguen estas universidades en temas de creación, identificando: sus orígenes, bases, métodos, y su razón de ser en la formación de profesionales en ciencias, ingeniería y humanidades. El hallazgo principal consistió en evidenciar como en los programas de formación profesional analizados, se aplica el pensamiento, el método y la estética propios del campo disciplinar del diseño, lo cual permite establecer los niveles de influencia de este campo del saber en áreas no concurrentes del diseño.

Palabras clave: pensamiento de diseño, métodos de diseño, estética, resolución de problemas, educación superior.

\begin{abstract}
This research article deals with the purpose of configuring a pattern, knowledge and practice of what is understood as design activities from its dimensions: thought, design methodology and aesthetic dimensions, within higher education teaching areas not associated to design professions. This research seeks to identify how teaching processes are understood and executed around the resolution of weakly structures problems in some other areas of knowledge and academic contexts of higher education in public universities in Bogotá, Colombia. Consequently, to achieve this goal, a description, characterization and analysis of the curricular contents that these universities follow in the creation of topics is developed by identifying their origins, bases, methods, and their reason of being in the formation of professionals in sciences, engineering and humanities. As a result, the main finding was to show how in the professional training programs analyzed, the thinking, method and aesthetics of the disciplinary field of design are applied, which allows to establish the levels of influence of this field of knowledge in non-concurrent areas of the design. As a result, the main finding on this study was to show how in the professional training programs analyzed, the thinking, method and aesthetics of the disciplinary field are applied, which allows to establish the levels of influence of this field of knowledge in non-concurrent areas of design.
\end{abstract}

Keywords: design thinking, design methodology, aesthetic, problem solving, higher education.

1 Diseñadora Industrial, Magíster en diseño en cerámica y vidrio, Universidad Autónoma de Colombia, Universidad Nacional de Colombia, Bogotá, Colombia. E-mail: Icagudelot@unal.edu.co

2 Diseñador Industrial, Magíster en pedagogía del diseño, Universidad Autónoma de Colombia, Bogotá, Colombia. E-mail: jlvelasco@ unal.edu.co 


\section{Introducción}

El creciente desarrollo de investigaciones asociadas a las disciplinas del diseño, ha puesto de manifiesto la necesidad de reflexionar en torno al diseño como campo de conocimiento. Lo mismo viene sucediendo con otras disciplinas creativas y proyectuales, siendo esta una discusión pertinente y vigente, que por su naturaleza epistemológica exige la identificación de una trama compleja de relaciones para llegar a establecer lo que puede o no ser considerado parte del campo disciplinar del diseño. Al respecto, Silva-Cañaveral, manifiesta:

(...) Es una tarea relativamente reciente a la que se han dado los/as investigadores en los campos creativos, al construir bancos con los enfoques y las perspectivas metodológicas aplicadas en el arte y el diseño; relacionar la investigación en estos campos disciplinares con conceptos presentes en las áreas de las ciencias naturales y sociales; proponer metodologías para dominios específicos; desarrollar competencias teóricas y disciplinares para la elaboración de proyectos; crear sistemas de análisis del pensamiento visual y modos de trabajo inter, multi y transdisciplinar. (Silva Cañaveral, 2016).

De este modo surgen también áreas de confluencia entre diferentes tipos de conocimiento, fenómeno al cual Zuluaga se refiere de la siguiente forma: "Los distintos tipos de conocimientos y saberes: sentido común, doxa legítima, técnicos, científicos y metateóricos están entrelazados, de modo que no se excluyen, sino que se complementan. Todos hacen parte del cuerpo cognitivo de la humanidad a partir del cual se comprende, interpreta o explica la realidad" (Zuluaga-Duque, 2017).

Así, establecer marcos de referencia desde el punto de vista del diseño, que relacionen de manera recíproca conocimientos y saberes de otros campos, permitirá expandir las fronteras de esta disciplina y ayudará a evidenciar que lo que se conci- be como pensamiento de diseño y los métodos y prácticas derivados de este, están presentes en diferentes áreas del saber especialmente cuando se desarrollan procesos de resolución de problemas.

En consecuencia, la investigación titulada: conocimiento y práctica del diseño en la formación de profesionales en la Universidad Colombiana, surge con el interés de abordar la problemática de cómo identificar las conexiones existentes entre pensamiento y práctica de diseño. Esto desde la perspectiva de lo entendido como diseño o sus actividades especializadas, dentro de las áreas de enseñanza universitaria no asociadas a programas de estudio en diseño o afines. En concordancia con esta inquietud, se formula la siguiente pregunta de investigación: ¿cómo y cuánto está influenciando el conocimiento y la práctica del diseño los contenidos y la formación de profesionales en Programas "no concurrentes" de diseño en la universidad colombiana?.

Para dar respuesta a la pregunta de investigación, se establece como hipótesis general que los métodos de resolución de problemas asociados al diseño, impartidos durante la formación a nivel de educación superior, están directamente influenciados por el pensamiento, el método y la estética del diseño. Estos últimos corresponden a los tres grandes ejes de análisis de la presente investigación, surgidos a partir del enfoque de autonomía del diseño como disciplina y profesión, que Aurelio Horta (2012), desarrolla en profundidad en su obra: trazos poéticos sobre el diseño. De este modo se comprobará que el pensamiento, la práctica y la estética del diseño tienen influencia en un amplio número de disciplinas, impactando positivamente el proceso de formación de profesionales en áreas tales como, ciencias, ingeniería y humanidades.

En consecuencia, se establecen como objetivos de la investigación: realizar un diagnóstico de cómo está influenciando el conocimiento y la práctica del diseño los contenidos y la formación de profesionales, en Programas "no concurrentes" del 
diseño en la universidad colombiana, primera etapa: Universidades públicas de Bogotá, Colombia; identificar en los contenidos curriculares de los Programas de formación profesional que se relacionen con procesos de pensamiento creativo, conceptos relacionados con el pensamiento, el método y la estética del diseño implícitos en la práctica y la resolución de problemas de diseño; y determinar el grado de influencia que tiene el proceso de diseño como estrategia pedagógica en la formación de profesionales en Programas"no concurrentes" del diseño en la universidad pública colombiana.

En las subsiguientes secciones de este escrito se hará referencia a los materiales y métodos empleados, la muestra analizada, y los resultados y conclusiones obtenidos tras analizar los contenidos de las asignaturas de cada programa de formación profesional y el grado de influencia del diseño en cada uno de los grupos profesionales establecidos.

\section{Materiales y métodos}

El tipo de investigación corresponde a la analítica o interpretativa, dado que se busca conocer más profundamente los niveles de influencia que el diseño, desde los tres ejes de análisis planteados, tiene en otras áreas del saber. Para ello se hace uso de herramientas como matrices de análisis, que permiten identificar pautas de relación no evidentes para ser usadas en investigaciones posteriores (Hurtado-de Barrera, 2010).
La recopilación de información se realiza inicialmente mediante la lectura interpretativa de autores que sustentan el marco teórico, luego se procede con el diseño de instrumentos y la posterior aplicación de los mismos para la recolección de datos. El análisis de la información se realizó a través de técnicas cualitativas y cuantitativas.

A nivel cualitativo se establecieron tres ejes principales, que cubren la epistemología del diseño evaluado en tres aspectos: el pensamiento, el método y la estética. Estos se soportan sobre lo expresado en las teorías de diversos autores respecto a los elementos de análisis. Así, Horta (2007), soporta el eje pensamiento; Bacon, Bürdek y Alexander, soportan el eje método (Read, 1961; Flusser, 2002; Munari, 2004; Goel, 2014); Calvera y Moholy-Nagy el eje estética (Moholy-Nagy, 1972; Bonsiepe, 1993; Camacho, 2009). La evaluación de estos parámetros en relación a los contenidos curriculares, se hizo mediante ponderación numérica.

El proceso de recolección de información consta de dos etapas: primero se realiza una síntesis de los aspectos teóricos principales de cada uno de los Programas profesionales elegidos para el análisis; y luego se hace una caracterización, analizando: objetivos, contenidos de las asignaturas y metodología de aula. Después se examinan estos contenidos desde los tres ejes de análisis definidos desde el marco teórico (pensamiento de diseño, método de diseño, y estética) y posteriormente se llevan estos datos a una herramienta de análisis denominada: marco de análisis de indicadores principales para la lectura y estudio de los programas de no diseño, ver tabla 1. 


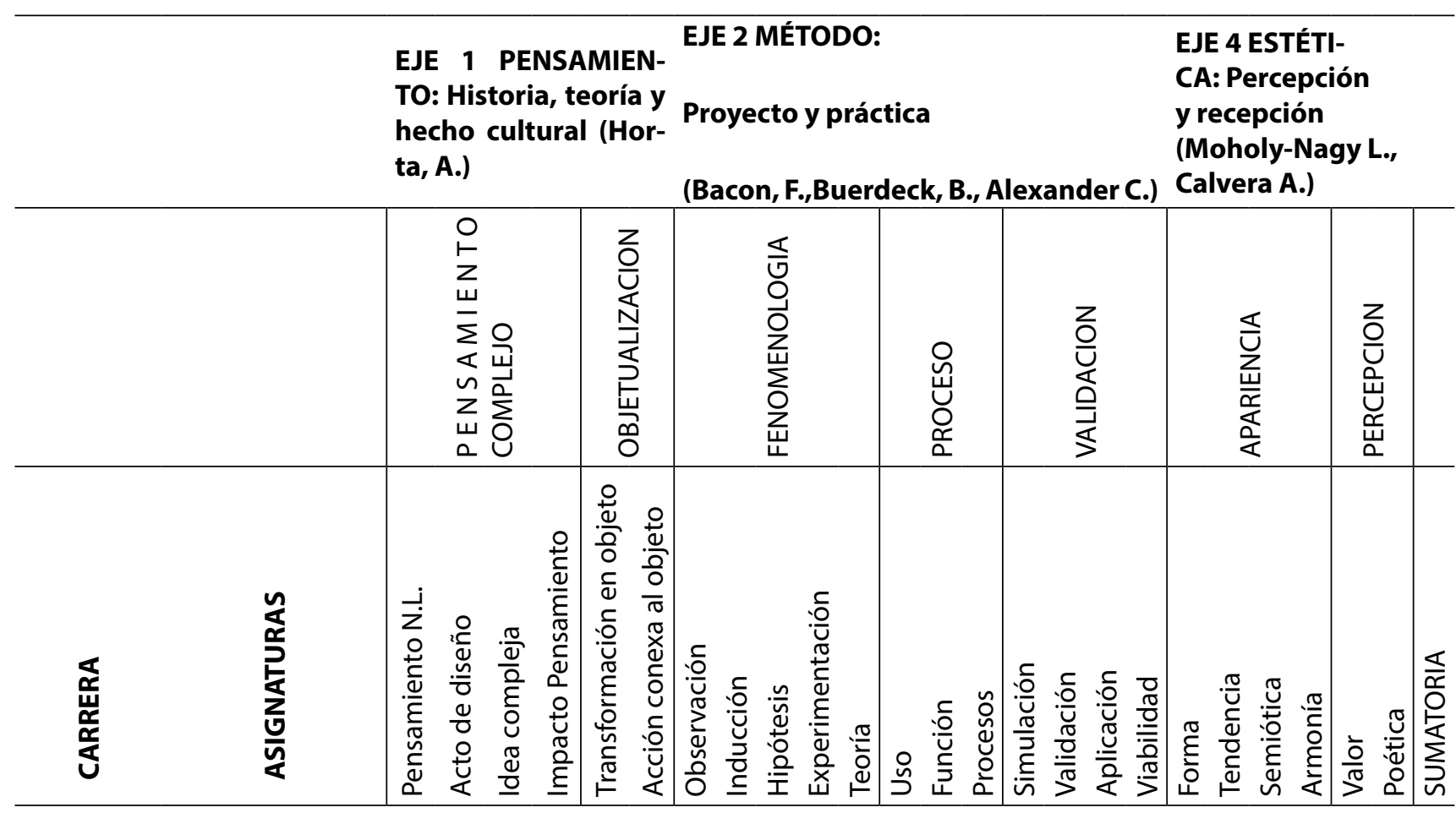

Tabla 1. Marco de análisis de indicadores principales para la lectura y estudio de los programas de no diseño.

En la herramienta TIPO MATRIZ DE LA TABLA 1, denominada: marco de análisis de indicadores principales para la lectura y estudio de los programas de no diseño, cada uno de los ejes está expresado en una serie de indicadores que se buscan en los contenidos de las asignaturas que presentan la palabra diseño, en su denominación o contenidos. Cuando la variable está presente, se califica con el valor uno, y cuando está ausente con cero. Luego se realiza la sumatoria, tanto en el eje vertical como en el horizontal, para conocer cuál es el eje que tiene mayor influencia y como se relacionan los que presentan influencia más débil.

Esta matriz permite tener una visión panorámica preliminar de cuáles son las áreas con las que con mayor frecuencia se relaciona el término diseño, y hacia cuál de los ejes tiende a aproximarse la idea del diseño como praxis.

El trabajo realizado durante los dos semestres de 2016, abarcó las universidades públicas de la ciudad de Bogotá, a saber: Universidad Nacional de
Colombia, UNAL, Universidad Distrital Francisco José de Caldas, UDFJC, y Universidad Pedagógica Nacional, UPN. En este artículo se presentan el proceso y resultados de las dos primeras, debido a las similitudes encontradas, en tanto que el principal volumen de la muestra correspondió a programas de ingeniería. Los resultados comparativos entre las tres Universidades serán objeto de otra publicación.

En la Universidad Nacional de Colombia, UNAL, se trabajó en veinte programas de formación profesional de diversas facultades, diferenciando claramente dos grandes grupos: los de ingeniería, que completaron diez programas, con 32 asignaturas relacionadas con diseño; y los de no ingeniería, que completaron otros diez, con doce asignaturas. Cabe acotar que los contenidos revisados corresponden a programas ofrecidos durante el año 2016. En la tabla 2 se presentan los programas por especialidad, con sus respectivas asignaturas. 


\begin{tabular}{|c|c|}
\hline \multicolumn{2}{|c|}{ Programas de ingeniería, Universidad Nacional de Colombia - Sede Bogotá } \\
\hline Programa & Asignaturas \\
\hline \multirow{6}{*}{$\begin{array}{l}\text { INGENIERIA MECATRÓNICA } \\
\text { INGENIERIA MECÁNICA } \\
\text { INGENIERIA QUIMICA }\end{array}$} & Diseño mecatrónico \\
\hline & Diseño de elementos de máquinas I \\
\hline & Diseño de elementos de máquinas II \\
\hline & Diseño, gestión y evaluación de proyectos. \\
\hline & Diseño de plantas y equipos \\
\hline & Diseño de procesos químicos y bioquímicos \\
\hline \multirow[t]{9}{*}{ INGENIERIA INDUSTRIAL } & $\begin{array}{l}\text { Modelos estocásticos para procesos de manufactura y } \\
\text { sistemas de servicios }\end{array}$ \\
\hline & Modelos y simulación \\
\hline & Taller de diseño de plantas \\
\hline & Taller de ergonomía e ingeniería de métodos \\
\hline & Taller de herramientas y problemas en ing. Industrial \\
\hline & Taller de invención y creatividad \\
\hline & Taller de ingeniería de la producción \\
\hline & Taller de metodología de la investigación \\
\hline & $\begin{array}{l}\text { Taller de simulación de procesos de manufactura y siste- } \\
\text { mas de servicios }\end{array}$ \\
\hline \multirow[t]{3}{*}{ INGENIERÍA AGRÍCOLA } & Diseño, gestión y evaluación de proyectos. \\
\hline & Diseño de estructuras de concreto \\
\hline & Diseño de sistemas de riego \\
\hline \multirow[t]{2}{*}{ INGENIERÍA AGRONÓMICA } & Diseño de experimentos \\
\hline & Diseño de experimentos* \\
\hline \multirow[t]{2}{*}{ INGENIERÍA CIVIL } & Diseño estructural \\
\hline & Diseño geométrico de vías \\
\hline \multirow[t]{4}{*}{ INGENIERÍA DE SISTEMAS } & Arquitectura de infraestructura \\
\hline & Elementos de computadores \\
\hline & Modelos y simulación \\
\hline & Taller de proyectos interdisciplinarios \\
\hline \multirow[t]{4}{*}{ INGENIERÍA ELÉCTRICA } & Análisis de sistemas de potencia \\
\hline & Taller de ingeniería eléctrica \\
\hline & Taller de proyectos interdisciplinarios \\
\hline & Instalaciones eléctricas \\
\hline \multirow[t]{2}{*}{ INGENIERÍA ELECTRÓNICA } & Taller de proyectos interdisciplinarios \\
\hline & Taller de ingeniería electrónica \\
\hline
\end{tabular}




\begin{tabular}{l|l}
\hline Programas de No Ingeniería, Universidad Nacional de Colombia - Sede Bogotá \\
\hline Programa & Asignaturas \\
\hline \multirow{2}{*}{ ADMINISTRACIÓN DE EMPRESAS } & Diseño y evaluación de proyectos \\
\cline { 2 - 2 } & Gerencia y gestión de proyectos \\
\hline BIOLOGÍA & Diseño de proyectos Antropología \\
\cline { 2 - 2 } & Diseño de proyectos en Arqueología \\
\hline GEOGRAFÍA & Diseño experimental \\
\hline FARMACIA & Laboratorio de investigación \\
\hline SICOLOGÍA & Diseño y desarrollo de productos farmacéuticos \\
\hline ECONOMÍA & Muestreo estadístico \\
\hline ESTADÍSTICA & Diseño, gestión y evaluación de proyectos \\
\hline CIENCIAS POLÍTICAS & Diseño y desarrollo de encuestas \\
\hline ZOOTECNIA & Diseño de investigación \\
\hline
\end{tabular}

Tabla 2. Programas con sus asignaturas, en la UNAL.

\begin{tabular}{l|l}
\hline Programas de Ingeniería, Universidad Distrital Francisco José de Caldas \\
\hline \multirow{2}{*}{ Programa } & Asignaturas \\
\hline \multirow{2}{*}{ INGENIERÍA MECÁNICA } & Programación orientada a objetos \\
\cline { 2 - 2 } & Diseño gráfico \\
\hline \multirow{4}{*}{ INGENIERIA INDUSTRIAL } & Diseño de elementos \\
\cline { 2 - 2 } & Diseño por elementos finitos \\
\cline { 2 - 2 } & Formulación y evaluación de proyectos \\
\cline { 2 - 2 } & Diseño de elementos normalizados \\
\hline & Taller de ergonomía \\
\cline { 2 - 2 } & Diseño industrial \\
\cline { 2 - 2 } & Diseño gráfico \\
\cline { 2 - 2 } & Taller de la producción \\
\cline { 2 - 2 } & Taller de diseño de la investigación \\
\cline { 2 - 2 } & Innovación empresarial \\
\cline { 2 - 2 } & Diseño integrado de producto y proceso \\
\cline { 2 - 2 } & Formulación y evaluación de proyectos \\
\cline { 2 - 2 } & Procesos industriales \\
\hline \multirow{2}{*}{ INGENIERÍA CIVIL } & Diseño estructuras \\
\cline { 2 - 2 } & Diseño y construcción de canales \\
\hline & Diseño de instalaciones \\
\cline { 2 - 2 } & Diseño y construcción de pavimentos \\
\cline { 2 - 2 } & Diseño geométrico de vías \\
\cline { 2 - 2 } & Formulación y evaluación de proyectos \\
\cline { 2 - 2 } &
\end{tabular}




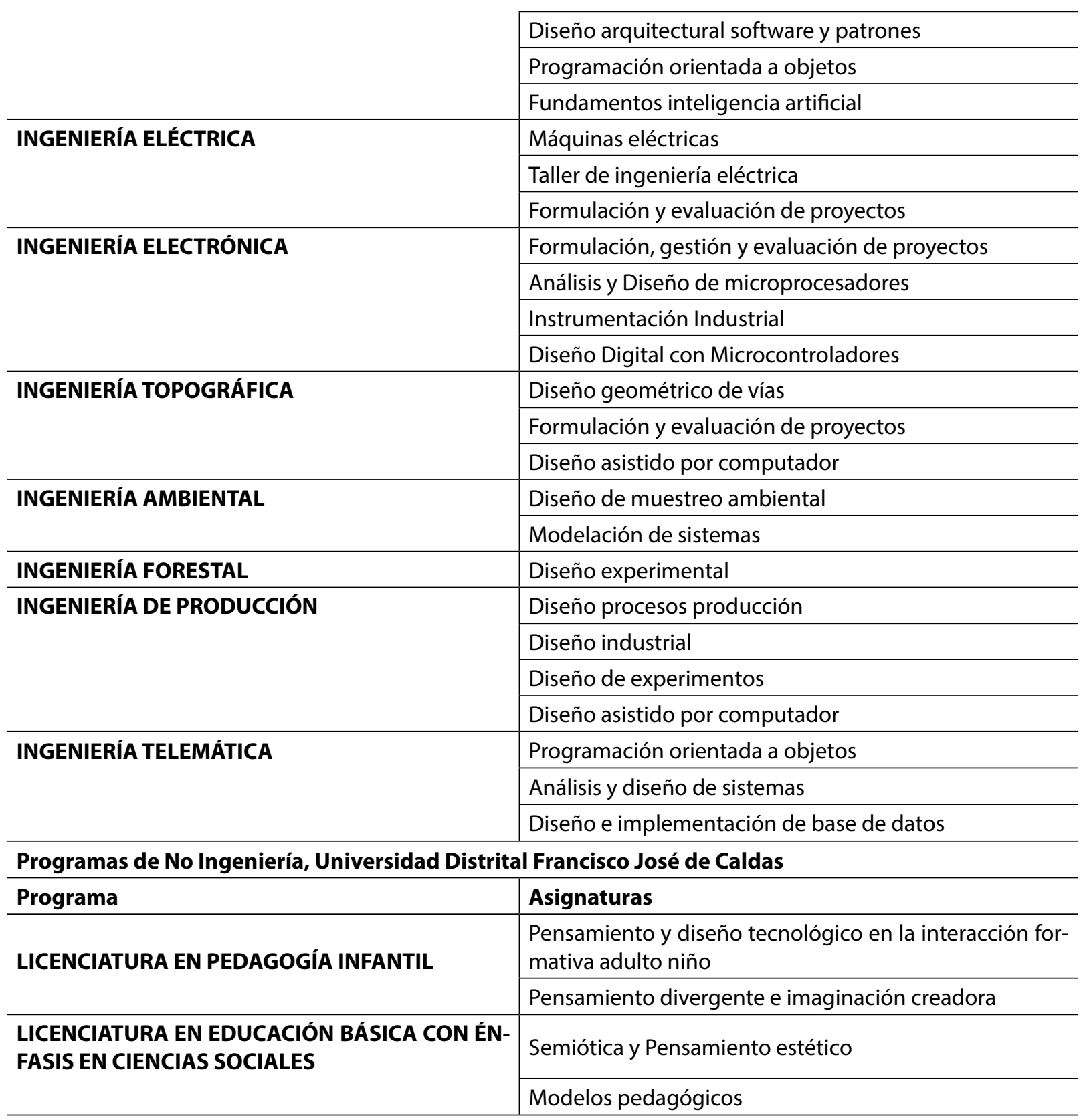

Tabla 3. Programas con sus asignaturas, en la UDFJC.

En la recolección de información y posterior análisis en la Universidad Distrital, se clasificó la muestra en un gran grupo de ingenierías y en un pequeño grupo de dos Programas de humanidades, ver tabla 3. Esto debido a que las otras áreas del conocimiento no tienen, actualmente, contenidos curriculares que cumplan con los requerimientos del filtro preliminar. 


\section{Resultados y discusión}

\subsection{Universidad Nacional de Colombia}

Ingenierías

En la UNAL se encontraron diez diferentes ingenierías que cumplían con los requisitos de contener en sus currículos asignaturas orientadas hacia el diseño. En los resultados del análisis, donde están desglosados los tres ejes principales de la investigación, con sus subcategorías e indicadores, se observa en el eje 1, pensamiento, que en la subcategoría de pensamiento complejo y objetualización, son pocos los contenidos curriculares en la UNAL que califican dentro de los mencionados indicadores.

En el eje 2, método, que pretende revisar los procesos metodológicos usados en el aula para la obtención de los resultados esperados, todas las asignaturas revisadas presentan un aporte importante en cuanto a fenomenología, que en esta investigación está relacionada con los cinco pilares heredados de la filosofía de Bacon, como son: observación, inducción, hipótesis, experimentación y teoría (Bonsiepe, 1993).

Los indicadores proceso y validación, están trazados para revisar mecanismos procedimentales y operativos al interior de cada cátedra. Se pone de manifiesto que las asignaturas dentro de casi la totalidad de Programas de los dos grupos: ingeniería y no ingeniería, presentan un importante acercamiento a los procesos como los tiene concebidos el diseño al respecto de proyecto y práctica.

En el eje 3, estética, existe una clara conjunción entre una problemática a resolver, un proceso y la obtención de un resultado solucionador. Se observan datos disímiles si se tiene en cuenta la subcategoría y su indicador, por ejemplo, la percepción y la apariencia no parecen ser prioridades en los proyectos de Ingeniería y tampoco de No Ingeniería.

\section{No Ingenierías}

Existen diez Programas por fuera de las ingenierías, seleccionados en la búsqueda de la influencia del diseño, cuyos contenidos se pueden considerar elementos de estudio en el presente análisis. Estos Programas son: Administración de empresas y Antropología con dos asignaturas, respectivamente; y Biología, Geografía, Farmacia, Sicología, Economía, Estadística, Ciencias Políticas y Zootecnia, con una asignatura cada uno.

Siguiendo la misma línea de análisis de los Programas de Ingeniería, en los Programas de No Ingeniería se determina que el eje 1, pensamiento, presenta una tendencia similar a las ingenierías; esto ya que los programas tabulados no evidencian contenidos amplios relacionados con las variables de este eje. El indicador Pensamiento no lineal es el que más sobresale, con 6 puntos de 12 posibles. En ese mismo eje, el indicador de transformación en objeto, es el que más bajas puntuaciones obtuvo. En vista de lo anterior, la sumatoria vertical en el eje de pensamiento no permite confirmar una aplicación importante de los conceptos estipulados por Horta (2012).

En el eje 2, método, cuatro indicadores alcanzan la puntuación máxima. Se evidencia entonces que al igual que en el grupo de Ingeniería, los Programas de No Ingeniería utilizan métodos prácticos y ordenados de planteamiento, seguimiento, evaluación y puesta a prueba de resultados.

Al revisar los puntajes del tercer eje, estética, solo tres indicadores obtienen puntuación: la forma, la tendencia y el valor. Se percibe aquí que la estética, al igual que en los grupos de Ingeniería, no cumple un papel fundamental para la resolución de problemas.

\subsection{Universidad Distrital Francisco José de Caldas}

\section{Ingenierías}

En el análisis del eje 2, método, se observa una tendencia semejante a la UNAL; es decir, las asignaturas revisadas presentan aportes significativos en cuanto a fenomenología, en los indicadores ob- 
servación, inducción, hipótesis, experimentación y teoría.

Los indicadores proceso y validación, igualmente se presentan de manera recurrente, evidenciándose así un importante acercamiento a los procesos metodológicos del diseño asociados al proyecto y la práctica.

En el eje 3, estética, en general se observa la poca presencia de los indicadores por lo cual se deduce que estos no son conceptos de alta relevancia, tanto en los proyectos de Ingeniería como en los de No Ingeniería.

\section{No Ingenierías}

En la revisión de los contenidos de los Programas de No Ingeniería, no se evidencia mayor desarrollo de conceptos determinados en el eje 1, pensamiento. Los Programas de licenciatura tienen establecido su trabajo de planteamiento de problemáticas en procesos personales subjetivos y socioculturales de los niños; esto hace más difícil establecer campos de aplicación más tangibles o materializables, en comparación con los que pudiera tener la ingeniería. En este sentido, la sumatoria vertical en el eje de pensamiento no permite evidenciar una aplicación sustancial de los conceptos tomados de Horta (2012).

En el eje 2, método, cinco de los doce indicadores establecidos alcanzan la totalidad de la puntuación máxima, 4 puntos. Estos son: observación, inducción e hipótesis, pertenecientes al subtema de fenomenología; y procesos, pertenecientes al subtema Proceso. Además, la validación, la aplicación y la viabilidad se encuentran presentes en aquellas asignaturas que aplican estos métodos como parte de un proceso de resolución de problemas.

Al revisar los puntajes del tercer eje, estética, los parámetros de poética y armonía, tienen un punto cada uno. Esta puntuación está específicamente asociada a la asignatura Semiótica y Pensamien- to estético. Los indicadores forma y semiótica, se encuentran relacionados tanto con esta asignatura como con Pensamiento divergente e imaginación creadora. En relación con estos puntajes, se puede evidenciar que la estética, al igual que en los grupos de Ingeniería, no cumple un papel fundamental en el proceso de resolución de problemas orientados hacia el pensamiento lógico. Sin embargo, cuando la noción de estética es totalmente explícita, como en el caso de la asignatura semiótica y pensamiento estético en la carrera de Licenciatura en educación básica con énfasis en ciencias sociales, o se plantean procesos de pensamiento divergente y creatividad, la importancia de este eje se incrementa notoriamente.

\subsection{Discusión}

El objetivo de esta investigación es poner de manifiesto que en la formación de estudiantes de pregrado, en las universidades públicas de Bogotá, en Programas diferentes a los especializados en diseño, existe un proceso de pensamiento, de método y uso de la estética que puede ser incluido dentro de los alcances que tiene el diseño, y que está resolviendo problemas del tipo con variantes o débilmente estructurados (Goel, 2014). Dentro de las ingenierías, se encuentran elementos comunes en la resolución de problemas a los que se usan frecuentemente en el diseño. El trabajo que se hace está orientado, tanto en ingeniería como en diseño, a desafiar al estudiante a plantear un marco de trabajo enfocado a la solución de problemas reales y que a través de una metodología sistemática alcance resultados de producto.

El Programa que más influencia tiene, en cuanto al diseño se refiere, es ingeniería industrial, con un total de 30 créditos en diseño lo cual significa que un $12.2 \%$ de los créditos están relacionados con el diseño. La carrera con menos créditos de diseño es ingeniería mecatrónica con un $1.3 \%$, respecto a toda su malla curricular. Esto aparentemente es un desbalance en cuanto a resultados, pero hay que tener en cuenta que ingeniería industrial tiene 9 asignaturas o microcurrículos en los que se 
evidencia, a través de la tabulación hecha en el marco de análisis de indicadores, que dicha ingeniería tiene asignaturas catalogadas como influenciadas por el diseño. Para una mejor comprensión del concepto de influencia, en el Marco de Análisis de la tabla 1, que se aplicó a todos los Programas de todas las universidades, se puede ejecutar una sumatoria vertical para detectar la influencia en los Programas de ingeniería y de no ingeniería en cada uno de los ejes, como se ilustra en la figura 1.

\section{UNIVERSIDAD NACIONAL - INGENIERÍA}

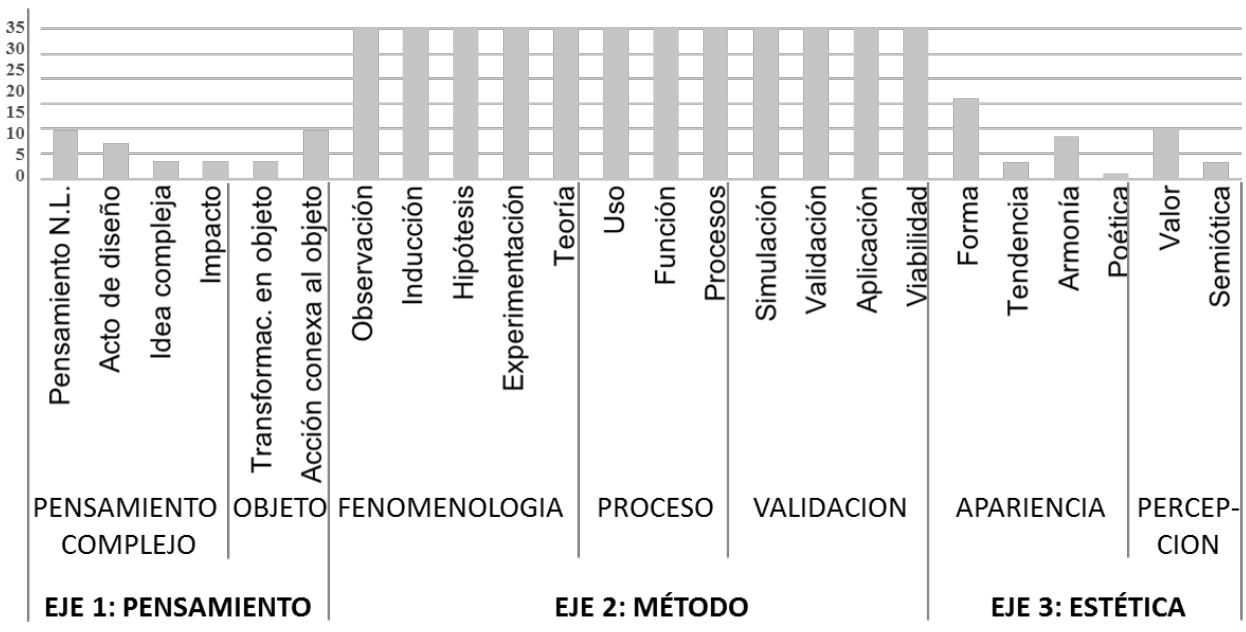

Figura 1. Influencia del diseño en programas de Ingeniería de la UNAL.

En comparación se tiene la figura 2, que representa la influencia del diseño en los Programas de no ingeniería de la UNAL.

UNIVERSIDAD NACIONAL - NO INGENIERÍA

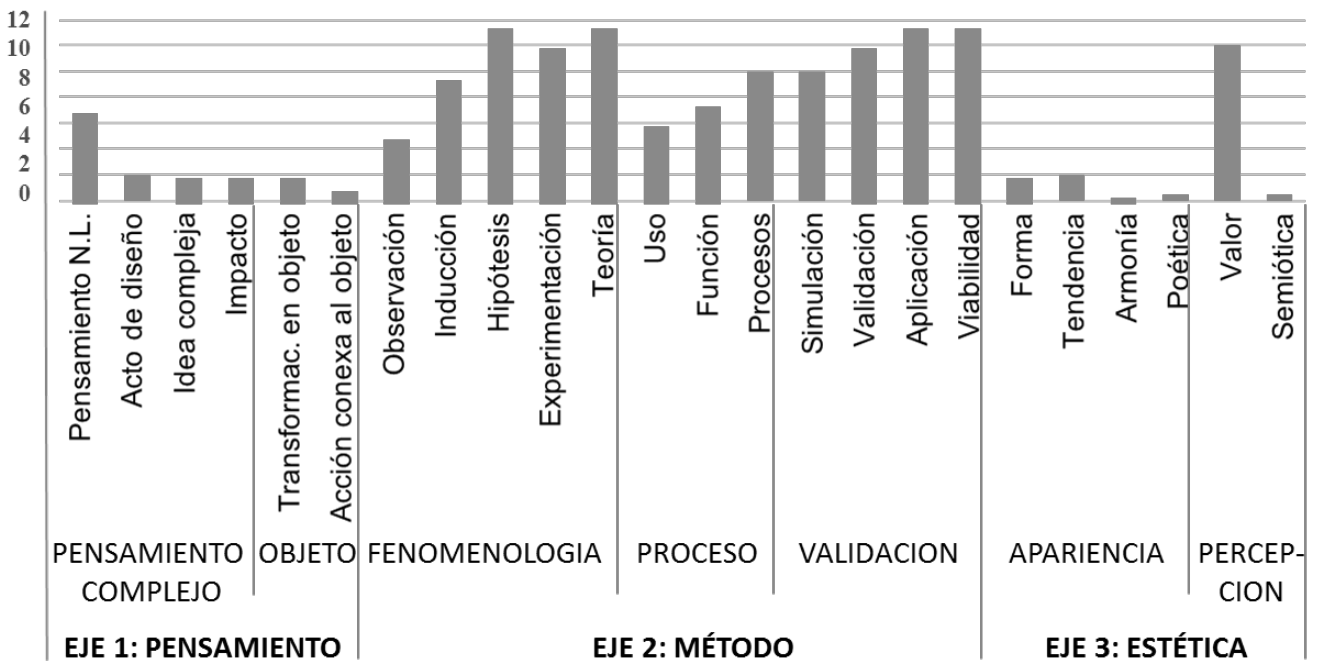

Figura 2. Influencia del diseño en programas de No ingeniería de la UNAL. 
La revisión de los elementos curriculares de los Programas en la UNAL, indica que, en ingeniería estos se orientan hacia el trabajo metodológico conducente a un resultado pragmático y funcional, respetando normas y parámetros físicos, mecánicos, eléctricos y de materiales, que alejan los conceptos de pensamiento y el resultado se aleja, también, de los conceptos de estética. En los Programas de No Ingeniería, la participación del eje de pensamiento tiene más importancia en los planteamientos; los métodos no se estandarizan en todas las asignaturas y definitivamente la esté- tica está más asociada al concepto de percepción del resultado final.

El análisis de la sumatoria vertical, en el marco de análisis de la Universidad Distrital, no se aleja mucho de lo evidenciado en la UNAL. Aunque en la Distrital hay más Programas de ingeniería, tiene menos asignaturas por carrera en promedio; sin embargo, la tendencia observada en la figura 3 es la misma, una importancia preponderante al eje del método.

COMPARATIVO U. NACIONAL Y U. DISTRITAL - INGENIERÍA

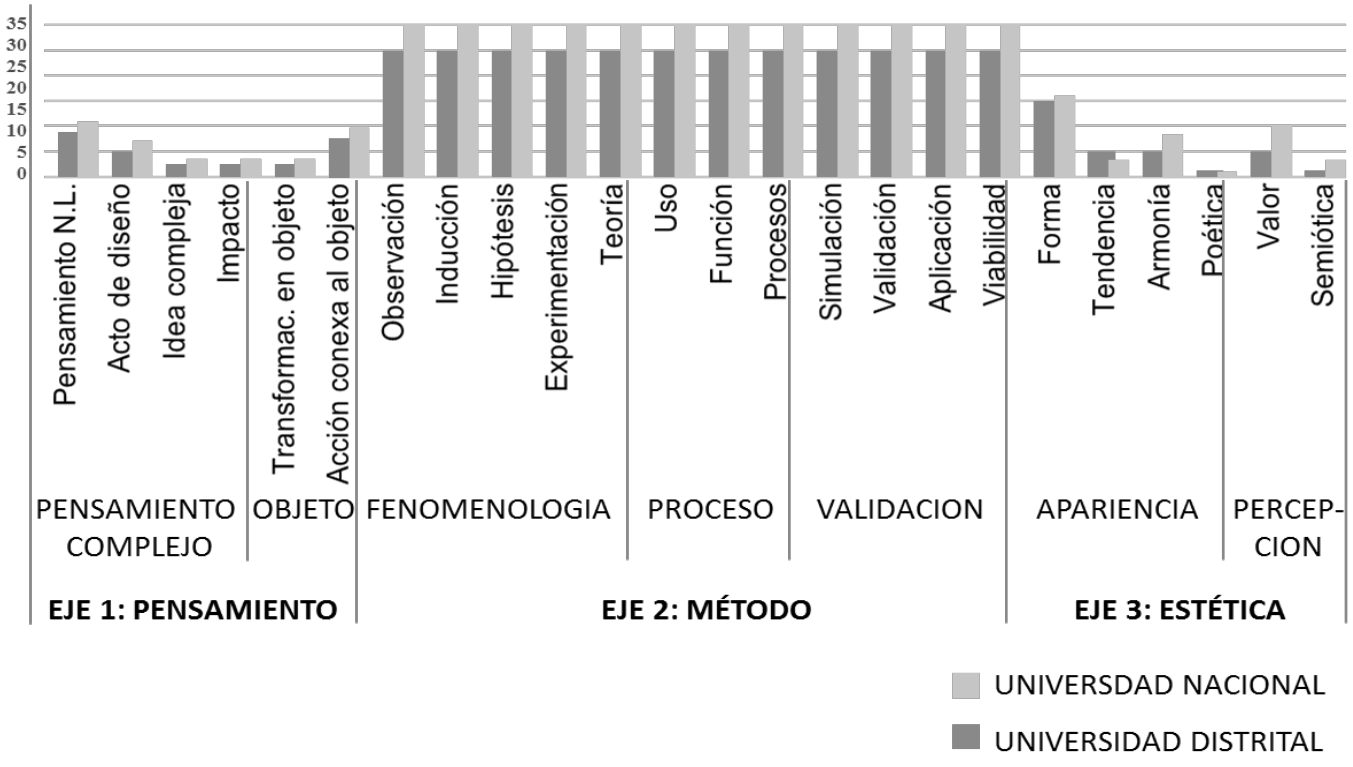

Figura 3. Análisis comparativo por ejes: programas de Ingeniería UNAL y UDFJC.

En la figura 3 se observa que los parámetros de fenomenología, proceso y validación pertenecientes al eje dos, tienen valores similares en ambas universidades. Se puede colegir que la educación en pregrado, en ambas universidades, tiene elementos metodológicos similares, en cuanto a la resolución de problemas para las ingenierías. 


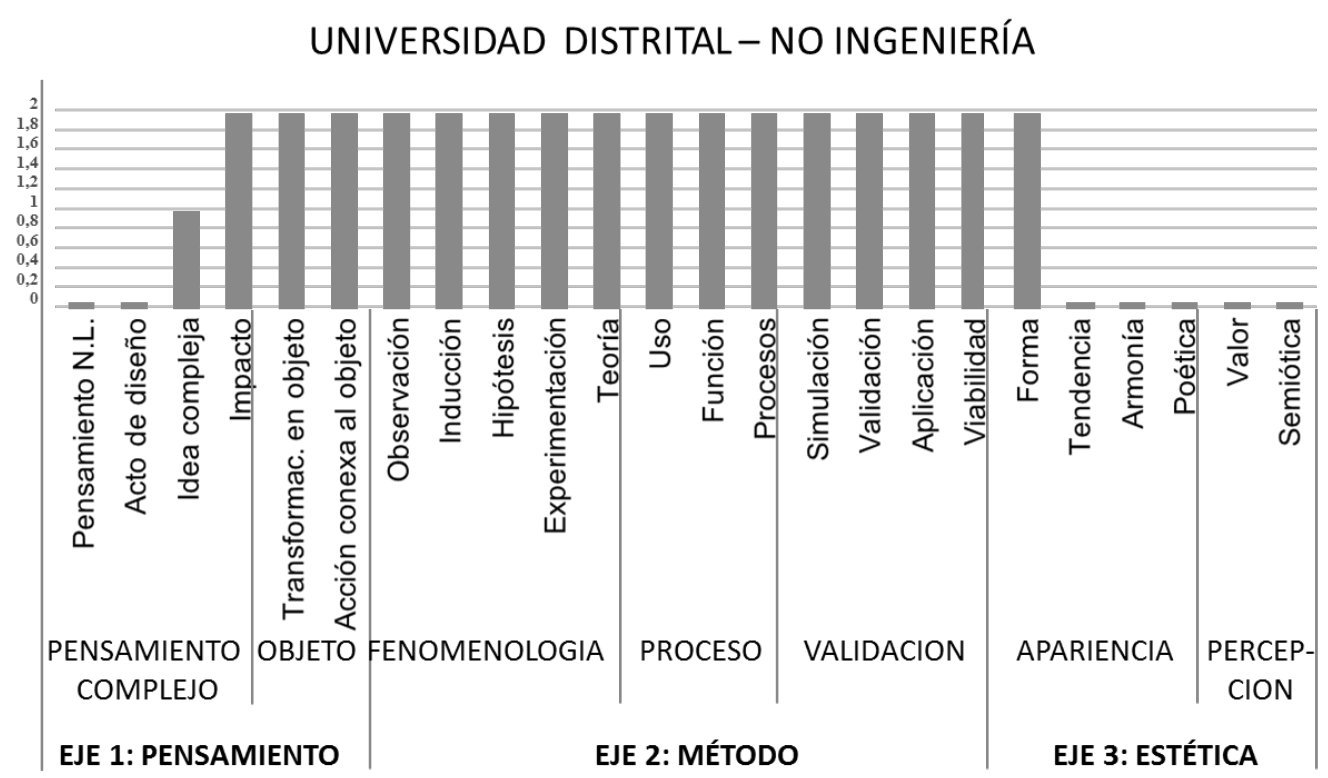

Figura 4. Influencia del diseño en No ingeniería, UDFJC.

En la figura 4 se puede apreciar que la tendencia de la influencia del diseño en carreras de No Ingeniería, se orienta de manera clara a los elementos preponderantes del eje dos, método. Sin embargo, surgen nuevos datos de los parámetros del eje uno, tales como impacto, transformación en objeto y acción conexa al objeto, que no es otra cosa que trabajo hecho con modelos de pensamiento aplicado de preparación y planeación. Estos modelos deben pasar luego a técnicas de recopilación, deducción y validación en el eje dos, el cual dentro de los puntajes obtenidos en el marco de análisis, tuvo la máxima calificación en todos los parámetros.

Tras analizar los contenidos de las asignaturas de cada carrera escogida como influenciada por el diseño, siguiendo una estructura jerárquica por área del conocimiento, microcurrículo y contenido, se pueden evidenciar hallazgos de alta relevancia para comprobar la hipótesis planteada. En primer lugar, se puede establecer que la influencia del conocimiento disciplinar de diseño no está dada principalmente por la inclusión de componentes microcurriculares relacionados con métodos de resolución de problemas que estimulen procesos de pensamiento no lineal, sino más bien por la aplicación de métodos que ayuden a la comprensión y delimitación del problema para después someter esta información a procesos de análisis a través del pensamiento lineal. Esto no significa que se le reste importancia a la fase creativa sino que tiene un peso y carácter diferente al que tiene para las profesiones asociadas propiamente al Diseño.

También se identificó que, si bien el trabajo de aula dentro de los Programas de ingeniería se establece con un esquema pedagógico diferente al trabajo de aula asociado a la formación en diseño, visto desde los tres ejes analizados, se puede determinar que hay elementos comunes en la resolución de problemas estructurados con los que se usan en el diseño, asociados principalmente al eje 2 , método. Por ejemplo, el uso de principios científicos e información técnica como parte del proceso de resolución de problemas, es una actividad que ambas áreas del saber comparten, aunque el procesamiento y análisis de esta información sea distinto. 
En el caso de la ingeniería, cuando se usa la matemática para aplicar la información de carácter técnico, los problemas se expresan simbólicamente y se resuelven de manera lógica, de modo que el resultado debe ser totalmente correcto. En el caso del diseño, la información técnica se traduce en determinantes y requerimientos del proyecto de diseño cuyas posibles respuestas o soluciones pueden ser muchas y no únicamente una exacta e indiscutible.

Adicionalmente, desde la Ingeniería, el proceso de resolución de problemas poco estructurados se desarrolla como una planeación de actividades con relación a una lógica de producción industrial, coincidiendo este planteamiento nuevamente con categorías analizadas desde el eje 2, método, tales como proceso y validación, y sus respectivos indicadores (uso, forma, proceso, simulación, aplicación y viabilidad).

La carrera que más influencia tiene del diseño, en los términos de esta investigación, es Ingeniería Industrial; en ella se identificaron nueve asignaturas cuyos contenidos convergen especialmente en lo relativo al método, y que se acerca a lo que se puede decir es la presencia potencial de escenarios pedagógicos similares entre los Programas especializados en diseño y dicha ingeniería.

La no linealidad del pensamiento, la presencia clara de un proceso y una validación, y la representación de resultados a través de una forma materializada, hacen de la ingeniería un área del saber influida claramente por el diseño. Para los Programas del grupo No ingeniería, el análisis muestra una ligera influencia de pensamiento, una gran influencia en el método y una baja influencia de estética.

\section{Conclusiones}

A manera de conclusión general, mediante la investigación se pudo comprobar que la metodología aplicada en diseño puede beneficiar de manera importante el desarrollo académico de las asignaturas analizadas, al incorporar modelos de pensamiento y procedimientos que pueden ampliar el marco de análisis y resolución de problemas de diferentes áreas del saber.

Desde una perspectiva holística, esta investigación permitió reconocer que existen interrelaciones importantes entre los saberes del diseño desde su no linealidad con saberes lineales, analíticos; esto debido a que el diseño, desde su pensamiento, método y praxis pone en diálogo distintos tipos de conocimiento en pro de erigir soluciones a problemas de diferente índole. Lo anterior indica el carácter integrador que el diseño como disciplina posee, y su potencial para propiciar reflexiones alternativas dentro o fuera del marco hegemónico del conocimiento científico.

\section{Agradecimientos}

Queremos agradecer al Sistema Unificado de Investigaciones, SUI, quien financió el proyecto a través de su convocatoria de Investigación número 24. También al Grupo de Investigación: Centro de Estudios Interdisciplinarios para el desarrollo, CEIDE, por su acompañamiento al proyecto. Igualmente, a la Universidad Nacional de Colombia, la Universidad Pedagógica Nacional y la Universidad Distrital Francisco José de Caldas, Instituciones que nos abrieron sus puertas para realizar el trabajo de campo.

\section{Referencias}

Bonsiepe, G. (1993). Las siete Columnas del Diseño. México D.F., México: UAM Azcapotzalco.

Camacho, T. (2009). Félix Beltrán en el diseño o el diseño en Félix Beltrán. Recuperado de: http:// www.artediez.es/articulos/camacho/beltran.pdf

Flusser, V. (2002). Filosofía del diseño. La forma de las cosas. Madrid, España: editorial Síntesis. 
Goel, V. (2014). Creative Brains. Designing in a real world. Frontiers in Human Neurscience, 8, 241 1-14. doi: 10.3389/fnhum.2014.00241.

Horta, A. (2007). Epistemología y Diseño. Notas críticas para una aproximación a la ciencia del diseño. Actas de Diseño 2. I Encuentro latinoamericano de Diseño. Diseño en Palermo. Argentina. 1 (2), 135-138.

Horta, A. (2012) Trazos poéticos sobre el diseño. Manizales, Colombia: Editorial Universidad de Caldas.

Hurtado-de Barrera, J. (2010). Metodología de la investigación. Guía para la comprensión holística de la ciencia. Bogotá, Colombia: Editorial Quirón-Sypal.

Moholy-Nagy, L. (1972) Nueva visión. Buenos Aires, Argentina: Ediciones Infinito.
Munari, B. (2004). Como nacen los objetos. México D.F, México: Gustavo Gili.

Read, H. (1961). Arte e Industria. Principios de Diseño Industrial. Primera edición. Buenos Aires, Argentina: Ediciones Infinito

Silva - Cañaveral, S. J. (2016). La investigación-creación en el contexto de la formación doctoral en diseño y creación en Colombia. Revista de Innovacion, Desarrollo e Innovación, 7 (1), 49 - 61. doi: https://doi.org/10.19053/20278306.v7.n1.2016.5601

Zuluaga - Duque, J. F. (2017). Relación entre conocimientos, saberes y valores: un afán por legitimar los saberes más allá de las ciencias. Revista de Investigación, Desarrollo e Innovación, 8 (1), 61 -76. doi: 10.19053/20278306.v8.n1.2017.5973 